\title{
Macroscopic quantum superpositon states of two-component Bose-Einstein condensates
}

\author{
Gábor Csire ${ }^{1,2, \text { f }}$ and Barnabás Apagyi ${ }^{1, \dagger}$ \\ ${ }^{1}$ Institute of Physics, Budapest University of Technology and Economics, \\ H-1111, Budafoki út 8, Hungary \\ ${ }^{2}$ Institute of Physics, Eötvös Loránd University, \\ H-1117, Pázmány Péter sétány 1/A, Hungary
}

(Dated: March 11, 2022)

\begin{abstract}
We examine a two-component Bose-Einstein condensate in a double-well potential. We propose a model for the creation of many-particle macroscopic quantum superposition states. The effect of dissipation on the formation of these states is also investigated with the Monte-Carlo wavefunction technique.

PACS numbers: 03.75.Gg, 03.75.Mn
\end{abstract}

\section{INTRODUCTION}

The principle of superposition is at the heart of quantum mechanics. Microscopic quantum superpositions are routinely observed in experiments, but for macroscopic objects the decoherence time is almost instantaneous [1, 2]. The famous thought experiment of Schrödinger [3] illustrates the problems caused by the transition from quantum to classical regime in the description of physical systems of increasing size. At the quantum-classical boundary, systems should be isolated long enough, so that creation of superposition of distinguishable states might be possible at least on mesoscopic scale. If quantum mechanics and classical mechanics are limiting cases of the same dynamics, the theory, which describes the relation between them, must be nonlinear and stochastic 4. Nonlinearity is crucial for the creation of superpositions of macroscopically distinguishable states, the so called macroscopic quantum superposition states (MQSS). These states were first suggested by Yurke and Stoler 5. A small number of experimental realizations has been reported. Friedman et al. 6] have shown that a SQUID can be put into a superposition of two magneticflux states: one corresponding to a few microamperes of current flowing clockwise, the other corresponding to the same amount of current flowing anticlockwise. Brune et al. 7, 8, produced superpositions involving microwave photons. MQSS with trapped ions have been created in Ref. 9]. Ourjoumtsev et al. [10] also demonstrated experimental MQSS using homodyne detection and photon number states as resources.

During the last decade ultracold gases became a very important testbed for many predictions of condensed matter physics [11. The underlying reason for this popularity is the high flexibility of the system parameters: the strength of interaction, the type and strength of exter-

\footnotetext{
*Electronic address: csire.gabor@wigner.bme.hu
}

${ }^{\dagger}$ Electronic address: apagyi@phy.bme.hu nal potential(s) the particles are moving in, or the phase difference between two (or more) condensates. This versatility allows us to check and examine, for example, predicted delicate phases in different dimensions, such as the Bardeen-Cooper-Shrieffer [12, 13] or the Mottinsulator phase [14 in 3D, the Berezinksii-KosterlitzThouless 15, 16] phase in 2D and the Tonks-Girardeau phase in 1D 17, 18. With the advent of Bose-Einstein condensates (BECs) a new experimental system has become available for the investigation of macroscopic quantum superposition states. Recently, several proposals for generation of MQSS in BECs have been reported in the literature. Cirac et al. 19] have provided a method which involves adiabatic transfer of a two-species condensate to the many-body ground state. Gordon and Savage [20, 21] use a two-component BEC with two-body interactions and weak Josephson coupling between the two components. In Ref. [22] a scheme of creating MQSS with scattering light from two BECs moving with opposite velocities has been proposed. MQSS of $\pi$-phase imprinted single component BEC have been predicted to exist in a double-well potential 23 .

In this study we work out a quasi-1D Hamiltonian model which describes two-component BEC states. In the two-mode approximation we solve the eigenvalue problems and construct MQSS (cat states) for small BEC systems appropriate for numerical treatment. By investigating phase decoherence and time development we establish signatures of cat states for larger BEC systems which develop into mixture states.

Section [II deals with the model, in section III we investigate MQSS in a double-well potential. Section IV] is left for treatment of decoherence and section $\mathrm{V}$ contains the conclusion and a summary.

\section{THE MODEL}

A two-component Bose-Einstein condensate can be described with the following second quantized Hamiltonian at zero-temperature: 


$$
H=H_{1}+H_{2}+H_{12}
$$

where

$$
\begin{gathered}
H_{i}=\int \mathrm{d}^{3} r\left(-\frac{\hbar^{2}}{2 m_{i}} \Psi_{i}^{\dagger}(\underline{r}) \Delta \Psi_{i}(\underline{r})+\Psi_{i}^{\dagger}(\underline{r}) V_{i}(\underline{r}) \Psi_{i}(\underline{r})\right) \\
+\frac{1}{2} g_{i i} \int \mathrm{d}^{3} r \Psi_{i}^{\dagger}(\underline{r}) \Psi_{i}^{\dagger}(\underline{r}) \Psi_{i}(\underline{r}) \Psi_{i}(\underline{r}) \\
H_{12}=g_{12} \int \mathrm{d}^{3} r \Psi_{1}^{\dagger}(\underline{r}) \Psi_{2}^{\dagger}(\underline{r}) \Psi_{1}(\underline{r}) \Psi_{2}(\underline{r})
\end{gathered}
$$

$V_{i}(\underline{r})$ is the external trapping potential. The coupling constants $g_{i i}$ and $g_{12}$ are the intra- and inter-species atom-atom interaction strengths:

$$
\begin{aligned}
g_{i i} & =\frac{4 \pi \hbar^{2}}{m_{i}} a_{i i} \\
g_{12} & =\frac{2 \pi \hbar^{2}}{m_{12}} a_{12}
\end{aligned}
$$

where $m_{12}$ denotes the reduced mass

$$
m_{12}=\frac{m_{1} m_{2}}{m_{1}+m_{2}},
$$

and $a_{i j}$ is the s-wave scattering length which can be tuned via Feshbach resonance. $\Psi_{i}^{\dagger}(\underline{r})$ and $\Psi_{i}(\underline{r})$ are the bosonic creation and annihilation operators for the two species satisfying the usual commutation rules and normalized to the number $N_{i}$ of particles of species $i$.

We will assume that the external potentials have the following form

$$
V_{i}(\underline{r})=\frac{1}{2} m_{i} \omega_{i, \perp}^{2}\left(y^{2}+z^{2}\right)+V_{i}(x),
$$

where

$$
V_{i}(x)=\frac{1}{2} m_{i} \omega_{i, x}^{2}\left(x^{2}+A \mathrm{e}^{-B x^{2}}\right) .
$$

We define

$$
\begin{array}{rlrl}
a_{i}^{L} & =\frac{a_{i}^{(0)}+a_{i}^{(1)}}{\sqrt{2}}, & a_{i}^{R}=\frac{a_{i}^{(0)}-a_{i}^{(1)}}{\sqrt{2}}, \\
\phi_{i}^{L}=\frac{\phi_{i}^{(0)}+\phi_{i}^{(1)}}{\sqrt{2}}, & \phi_{i}^{R}=\frac{\phi_{i}^{(0)}-\phi_{i}^{(1)}}{\sqrt{2}},
\end{array}
$$

where $\phi_{i}^{(0)}$ and $\phi_{i}^{(1)}$ are the mean-field ground and first excited states for an atom of species $i$, and they are normalized to one. $a_{i}^{(0)}$ and $a^{(1)}$ are the annihilation operators in the ground and excited state, respectively and satisfy the usual commutation relations. $\phi_{i}^{(0)}$ and $\phi_{i}^{(1)}$ can be obtained from the coupled Gross-Pitaevskii (GP) equations [24, 25]. The confinement of the potential is relatively strong in the radial directions, therefore, one may assume that the evolution in the weak x-direction decouples from that of in the strong [yz]-plane. Using these simplifying assumptions the three-dimensional equation can be reduced to the following coupled, timeindependent, one-dimensional equations [26]

$$
\begin{aligned}
& \mu_{1} \phi_{1}=\left[-\frac{1}{2} \partial_{x x}+\widetilde{V}_{1}(x)+b_{11}\left|\phi_{1}\right|^{2}+b_{12}\left|\phi_{2}\right|^{2}\right] \phi_{1}, \\
& \mu_{2} \phi_{2}=\left[-\frac{\kappa}{2} \partial_{x x}+\frac{1}{\kappa} \widetilde{V}_{2}(x)+b_{21}\left|\phi_{1}\right|^{2}+b_{22}\left|\phi_{2}\right|^{2}\right] \phi_{2},
\end{aligned}
$$

where $\mu_{1}$ and $\mu_{2}$ are the chemical potentials and

$$
\widetilde{V}_{i}(x)=\frac{\lambda_{i}^{2}}{2}\left(x^{2}+\widetilde{A} \mathrm{e}^{-\widetilde{B} x^{2}}\right),
$$

$b_{11}=2 a_{11} N_{1}, b_{22}=2 a_{22} N_{1} \kappa / \gamma, b_{12}=b_{21}=2 a_{12} N_{1}(1+$ $\kappa) /(1+\gamma), \gamma=\omega_{2, \perp} / \omega_{1, \perp}, \kappa=m_{1} / m_{2}, \lambda_{1}=\omega_{1, x} / \omega_{1, \perp}$, $\lambda_{2}=\omega_{2, x} / \omega_{1, \perp}, \widetilde{A}=A / a_{0}^{2}$, and $\widetilde{B}=B a_{0}^{2}$. The distances are measured in units $a_{0}=\sqrt{\hbar / m_{1} \omega_{1, \perp}}$. The normalization is such that $\int\left|\phi_{1}\right|^{2} \mathrm{~d} x=1$ and $\int\left|\phi_{2}\right|^{2} \mathrm{~d} x=N_{2} / N_{1}$. Moreover, the relation $\gamma^{2}=\kappa$ must hold if both species experience the same $\mathrm{HO}$ potential.

According to the two-mode approximation [27] we expand the field operator as follows

$$
\Psi_{i}=\left[a_{i}^{L} \phi_{i}^{L}(x)+a_{i}^{R} \phi_{i}^{R}(x)\right] \chi_{i, \perp}(y, z),
$$

where $\chi_{i, \perp}$ satisfies the corresponding 2D $\mathrm{HO}$ Schrödinger equation. It is convenient to introduce angular momentum operators [28]

$$
\begin{aligned}
J_{i}^{x} & =\frac{1}{2}\left[\left(a_{i}^{R}\right)^{\dagger} a_{i}^{L}+\left(a_{i}^{L}\right)^{\dagger} a_{i}^{R}\right], \\
J_{i}^{y} & =\frac{-\mathrm{i}}{2}\left[\left(a_{i}^{R}\right)^{\dagger} a_{i}^{L}-\left(a_{i}^{L}\right)^{\dagger} a_{i}^{R}\right], \\
J_{i}^{z} & =\frac{1}{2}\left[\left(a_{i}^{R}\right)^{\dagger} a_{i}^{R}-\left(a_{i}^{L}\right)^{\dagger} a_{i}^{L}\right],
\end{aligned}
$$

where $J_{i}^{x}, J_{i}^{y}$ correspond to the correlation between the two wells and $J_{i}^{z}$ is the particle number difference between the two wells for the species $i$. Furthermore, $J_{i}^{2}$ is the Casimir invariant of the $\mathrm{SU}(2)$ algebra:

$$
J_{i}^{2}=\left(J_{i}^{x}\right)^{2}+\left(J_{i}^{y}\right)^{2}+\left(J_{i}^{z}\right)^{2}=\frac{N_{i}}{2}\left(\frac{N_{i}}{2}+1\right) .
$$

By substituting (12) into (1) gives the following result within the improved two-mode approximation [29, 30]

$$
\begin{aligned}
H & =\sum_{i=1}^{2}\left[\left(\alpha_{i}+\epsilon_{i}\right) J_{i}^{x}+\beta_{i}\left(J_{i}^{x}\right)^{2}+\gamma_{i}\left(J_{i}^{z}\right)^{2}\right] \\
& +\alpha_{12} J_{1}^{x}+\alpha_{21} J_{2}^{x}+\beta_{12} J_{1}^{x} J_{2}^{x}+\gamma_{12} J_{1}^{z} J_{2}^{z}
\end{aligned}
$$

where 


$$
\begin{aligned}
\epsilon_{i} & =-\frac{2}{a_{0}} \int \mathrm{d} x\left[\frac{\hbar^{2}}{2 m}\left(\nabla \phi_{i}^{L}\right)\left(\nabla \phi_{i}^{R}\right)+\left(V(x)+\hbar \omega_{i, \perp}\right) \phi_{i}^{L} \phi_{i}^{R}\right] \\
\alpha_{i} & =\left(N_{i}-1\right) g_{i i} \frac{m_{i} \omega_{i, \perp}}{2 a_{0} \pi \hbar} \int \mathrm{d} x\left[\left(\phi_{i}^{R}\right)^{3} \phi_{i}^{L}+\left(\phi_{i}^{L}\right)^{3} \phi_{i}^{R}\right] \\
\alpha_{i j} & =-\frac{2}{a_{0} \pi \hbar} \frac{N_{j}}{N_{i}} g_{12} \frac{m_{1} \omega_{1, \perp} m_{2} \omega_{2, \perp}}{m_{1} \omega_{1, \perp}+m_{2} \omega_{2, \perp}} \int \mathrm{d} x\left[\left(\phi_{i}^{R}\right)^{2} \phi_{j}^{R} \phi_{j}^{L}+\left(\phi_{i}^{L}\right)^{2} \phi_{j}^{R} \phi_{j}^{L}\right] \\
\beta_{i} & =g_{i i} \frac{m_{i} \omega_{i, \perp}}{a_{0} \pi \hbar} \int \mathrm{d} x\left(\phi_{i}^{L}\right)^{2}\left(\phi_{i}^{R}\right)^{2} \\
\beta_{12} & =\frac{4 g_{12}}{a_{0} \pi \hbar} \frac{m_{1} \omega_{1, \perp} m_{2} \omega_{2, \perp}}{m_{1} \omega_{1, \perp}+m_{2} \omega_{2, \perp}} \int \mathrm{d} x \phi_{1}^{L} \phi_{1}^{R} \phi_{2}^{L} \phi_{2}^{R}, \\
\gamma_{i} & =g_{i i} \frac{m_{i} \omega_{i, \perp}}{8 a_{0} \pi \hbar} \int \mathrm{d} x\left[\left(\phi_{i}^{L}\right)^{2}-\left(\phi_{i}^{R}\right)^{2}\right]^{2}, \\
\gamma_{12} & =\frac{g_{12}}{a_{0} \pi \hbar} \frac{m_{1} \omega_{1, \perp} m_{2} \omega_{2, \perp}}{m_{1} \omega_{1, \perp}+m_{2} \omega_{2, \perp}} \int \mathrm{d} x\left[\left(\phi_{1}^{L}\right)^{2}-\left(\phi_{1}^{R}\right)^{2}\right]\left[\left(\phi_{2}^{L}\right)^{2}-\left(\phi_{2}^{R}\right)^{2}\right] .
\end{aligned}
$$

We measure the mass in units of the unified atomic mass unit.

A similar model was introduced within a simple twomode approach in Ref. 31.

\section{MACROSCOPIC QUANTUM SUPERPOSITON STATES}

In this section we want to determine the domain of $a_{i j}$ 's where it is possible to create cat states. We solve the eigenvalue problem of the Hamiltonian (15). The state space can be spanned in the $\left(N_{1}+1\right)\left(N_{2}+1\right)$ Fock state basis $\left|n_{1}^{L}, N_{1}-n_{1}^{L}\right\rangle_{1}\left|n_{2}^{L}, N_{2}-n_{2}^{L}\right\rangle_{2}$, where $n_{i}^{L}$ is the number of particles of species $i$ in the left well. An arbitrary state reads as follows

$$
|\Phi\rangle=\sum_{n_{1}^{L}=0}^{N_{1}} \sum_{n_{2}^{L}=0}^{N_{2}} c_{n_{1}^{L}, n_{2}^{L}}\left|n_{1}^{L}, N_{1}-n_{1}^{L}\right\rangle_{1}\left|n_{2}^{L}, N_{2}-n_{2}^{L}\right\rangle_{2},
$$

where

$$
\begin{aligned}
& \left|n_{1}^{L}, N_{1}-n_{1}^{L}\right\rangle_{1}\left|n_{2}^{L}, N_{2}-n_{2}^{L}\right\rangle_{2}= \\
& {\left[\prod_{i=1}^{2} \frac{\left(\left(a_{i}^{L}\right)^{\dagger}\right)^{n_{i}^{L}}}{\sqrt{n_{i}^{L} !}} \frac{\left(\left(a_{i}^{R}\right)^{\dagger}\right)^{N_{i}-n_{i}^{L}}}{\sqrt{\left(N_{i}-n_{i}^{L}\right) !}}\right]|0,0\rangle_{1}|0,0\rangle_{2} .}
\end{aligned}
$$

To obtain the parameters appearing in the Hamiltonian 15. we need to determine the mean-field ground and first excited states $\phi_{i}^{(0)}$ and $\phi_{i}^{(1)}$ from the time-independent coupled Gross-Pitaevskii equations 10a- 10b. This can be done in case of the ground state $\phi_{i}^{(0)}$ by using the imaginary time evolution method 32 combined with the split-step operator technique [33. For getting the first excited state $\phi_{i}^{(1)}$ we use the shooting method [34].
We define $P$ as the number $N_{\text {cat }}$ of superpositions of two distinguishable states (associated with the cat states or MQSS) divided by the total number $N_{t o t}=\left(N_{1}+\right.$ 1) $\left(N_{2}+1\right)$ of eigenstates ordered in sequence of growing eigenenergies,

$$
P=\frac{N_{c a t}}{N_{t o t}}
$$

In practice the selection of cat states proceeds via the search of the $N_{t o t}$ states (obtained by diagonalisation of (15)) for those members which possess two expansion coefficients $\left|c_{n_{1}^{L}, n_{2}^{L}}\right|^{2}$ greater than 0.4 .

For our numerical investigation we fix the following parameters: $m_{1}=m_{2}=87, \omega_{1, \perp}=\omega_{2, \perp}=2 \pi \times 710 \mathrm{~Hz}$, $\lambda_{1}=\lambda_{2}=0.2, \widetilde{A}=50, \widetilde{B}=10$. This means that our exploratory investigation for two-component cat states will be carried out for the case of a quasi onedimensional (cigar-like) ${ }^{87} \mathrm{Rb}$ Bose gas where the two components correspond to two hyperfine states of the rubidium atom. Such two-component BEC systems are routinely produced in laboratories 35. with particle numbers $N_{1} \sim N_{2} \sim 10000$. But simulating such a great number of atoms in the gas is beyond the capacity of today's computers. We think, however, that features of cat states to be presented here for lower particle numbers $N_{1}$ and $N_{2}$ will be valid also for larger $N_{i}$ values. Therefore we shall carry out the calculations at particle numbers $N_{1}, N_{2} \sim 1-20$ and vary the scattering lengths in the possible domain which can be achieved by using the Feshbach resonance method 36.

In Fig. 1 the probability $P$ of cat state formation is shown as function of $a_{11}$ and $a_{12}$ with fixed parameters $a_{22}=2 \mathrm{~nm}$, and particle numbers $N_{1}=4$ and $N_{2}=30$. We see an almost symmetrical triangle which is cut at $a_{12}=0$ where there is no two-component cat states. This is reasonable because the components are decoupled and, at the most, one-component cat states can be 


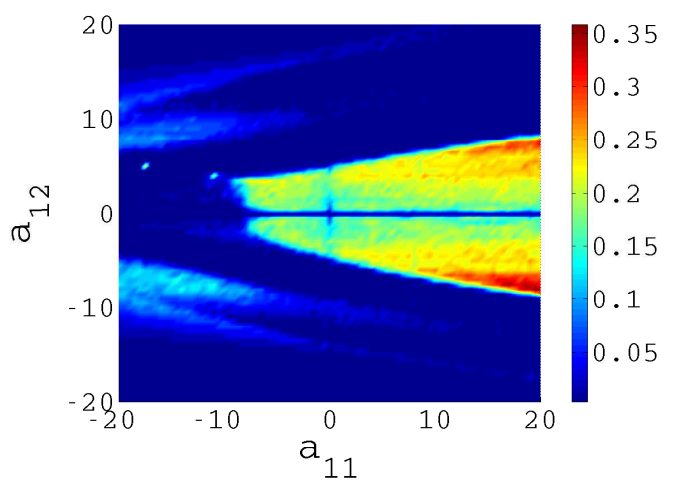

FIG. 1: (Color online) $P$ as function of $a_{11}$ and $a_{12}$ in nm; fixed parameters: $N_{1}=4, N_{2}=30, a_{22}=2 \mathrm{~nm}$.

formed with such parameter values. We observed however also that there is no two-component cat states when the sign of $a_{22}$ is changed to negative (at the above fixed parameters).

Therefore, it is reasonable to study the formation of cat states as function of the particle numbers $N_{1}, N_{2}$.

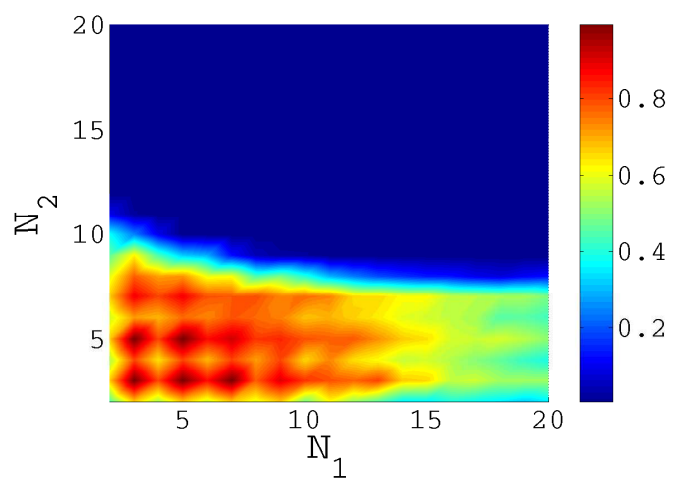

FIG. 2: (Color online) $P$ as function of $N_{1}$ and $N_{2}$; fixed parameters: $a_{11}=2 \mathrm{~nm}, a_{12}=4 \mathrm{~nm}, a_{22}=-2 \mathrm{~nm}$.

In Fig. $2 P$ is shown as function of $N_{1}$ and $N_{2}$ at positive $a_{11}, a_{12}$ and negative $a_{22}$ values. A sharp horizontal border can be observed from $N_{2}>10$, independent of $N_{1}$. This result explains the former finding of no cat state when $a_{22}$ has been changed to negative values. We numerically observed also that this horizontal phase separation border (between cat states and no cat states) can be raised to larger $N_{2}$ values by raising also the value of $a_{22}$.

In Fig. 3(a) all the scattering lengths have been fixed at positive values. It can be seen that the probability $P$ of forming cat states is lowered at growing particle numbers (decline is slower for component with smaller scattering length). But this means only that greater particle numbers involve more $N_{\text {tot }}$ states and thus a smaller fraction becomes cat states. This can be visualized if the result

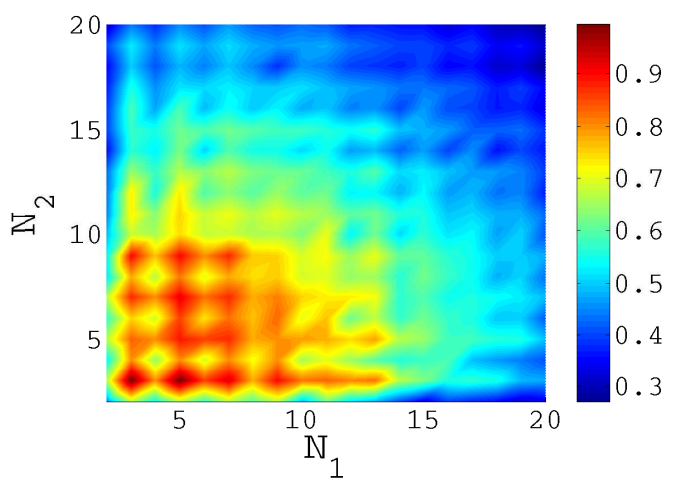

(a)

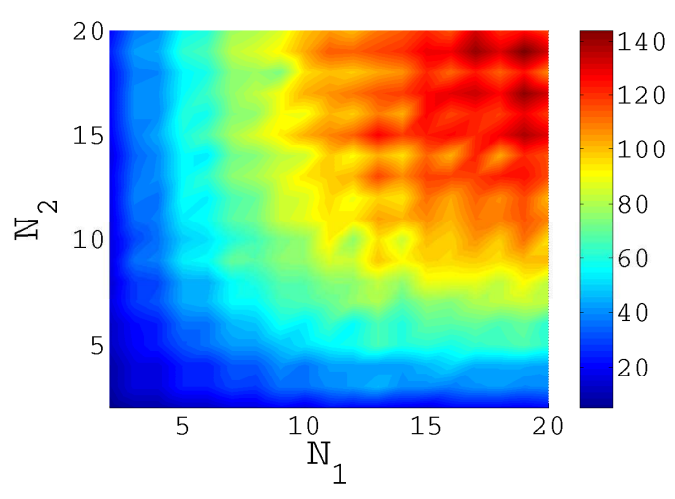

(b)

FIG. 3: (Color online) Fixed parameters: $a_{11}=2 \mathrm{~nm}, a_{12}=$ $4 \mathrm{~nm}, a_{22}=3 \mathrm{~nm}$. (a) $P$ as function of $N_{1}$ and $N_{2}$. (b) $N_{\text {cat }}$ as function of $N_{1}$ and $N_{2}$.

of the same calculation is shown not for the probability $P$ but for the number $N_{\text {cat }}$ of cat states. In Fig. 3(b) we see that the number of cat states are growing up to 140 at particle numbers $N_{1}=N_{2}=20$ which means however total number of states $N_{t o t}=441$, and therefore gives for $P$ only the value 0.3 which has been represented by blue color in Fig. 3(a).

In Fig. 4(a) the intra-species scattering lengths $a_{11}, a_{22}$ are positive while the inter-species scattering length $a_{12}$ is negative. According to the color bars we see that $P$ decreases here more strongly compared to Fig. 3(a) but the number of cat states is also decreasing, as can be seen in Fig. 4(b) where $N_{\text {cat }}$ is visualized for the same set of parameters.

In Fig. 5(a) and Fig. 5(b) all scattering lengths take negative values. We see that for larger particle numbers there are no cat states at all.

\section{DECOHERENCE}

We study the effect of dissipation. The dominant source is the thermal cloud of noncondensed atoms. 


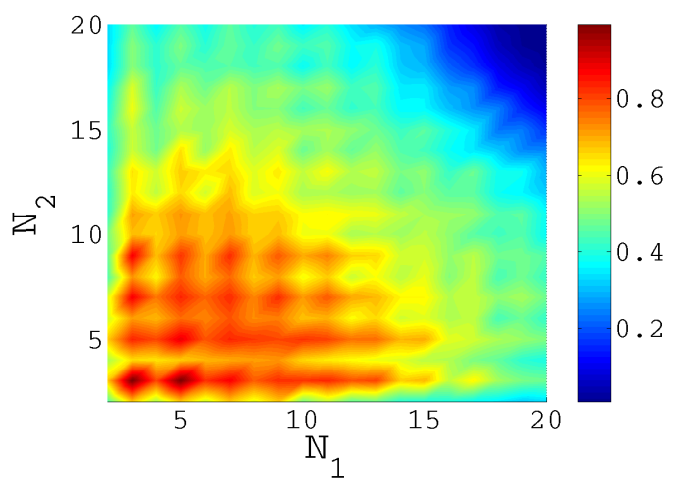

(a)

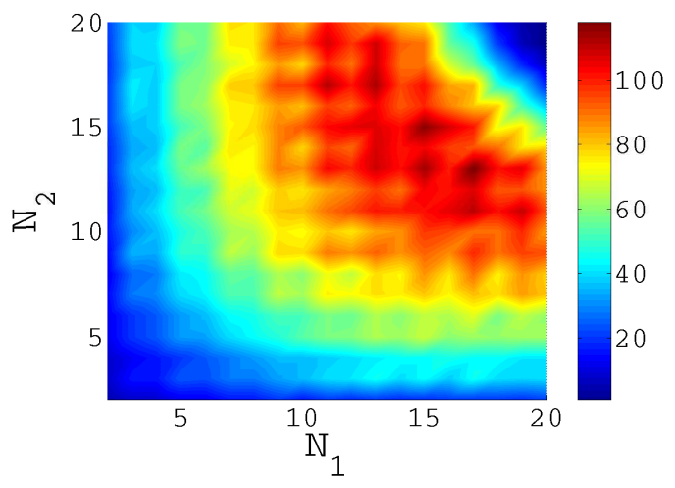

(b)

FIG. 4: (Color online) Fixed parameters: $a_{11}=2 \mathrm{~nm}, a_{12}=$ $-4 \mathrm{~nm}, a_{22}=3 \mathrm{~nm}$. (a) $P$ as function of $N_{1}$ and $N_{2}$. (b) $N_{\text {cat }}$ as function of $N_{1}$ and $N_{2}$.

Other possible sources are ambient magnetic fields, different scattering lengths, three-body losses 37. We will only investigate the effect of elastic two-body interactions between the noncondensed and condensed atoms. This type of interaction preserves the number of condensed atoms and leads to phase-damping which dominates for MQSS 21].

The most general quantum mechanical Markovian master equation has the form 38

$$
\frac{\partial}{\partial t} \rho=-\frac{\mathrm{i}}{\hbar}[H, \widehat{\rho}]+\sum_{\alpha}\left[\mathcal{L}_{\alpha} \rho \mathcal{L}_{\alpha}^{\dagger}-\frac{1}{2}\left\{\mathcal{L}_{\alpha}^{\dagger} \mathcal{L}_{\alpha}, \rho\right\}\right]
$$

where $\rho$ is the density matrix of system states, $\mathcal{L}_{\alpha}$ 's are the Lindblad operators containing system-environmental interaction, and $\{\cdot, \cdot\}$ is the anticommutator. The Lindblad formalism is valid when the coupling between the system and environment is weak 39. We use the following Lindblad generators to describe the phase-damping:

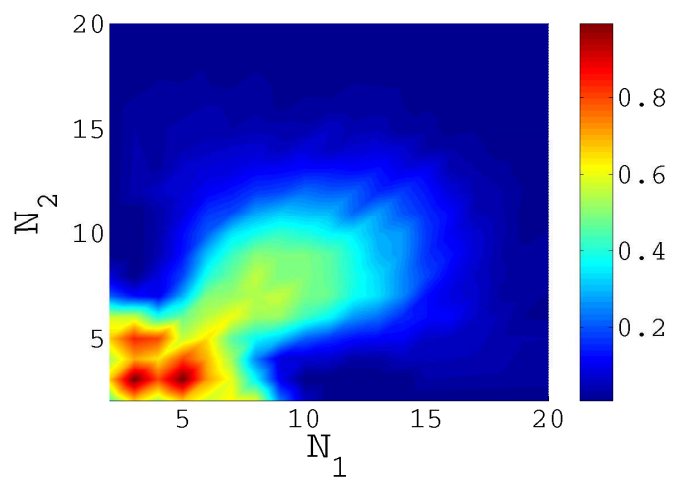

(a)

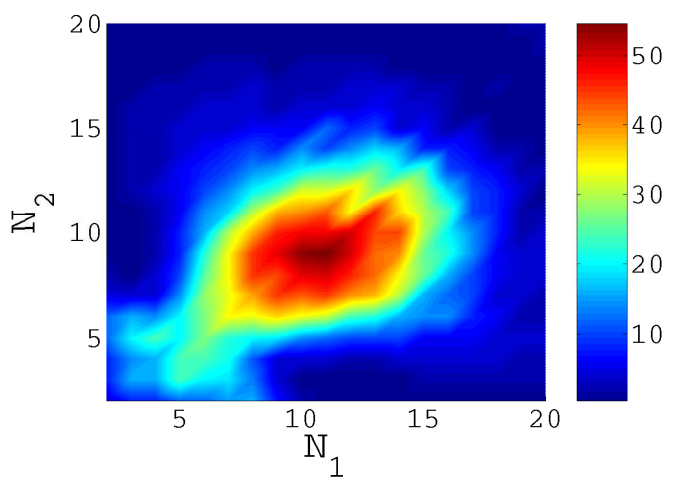

(b)

FIG. 5: (Color online) Fixed parameters: $a_{11}=-2 \mathrm{~nm}, a_{12}=$ $-4 \mathrm{~nm}, a_{22}=-3 \mathrm{~nm}$. (a) $P$ as function of $N_{1}$ and $N_{2}$. (b) $N_{\text {cat }}$ as function of $N_{1}$ and $N_{2}$.

$$
\begin{aligned}
& \mathcal{L}_{1}=\sqrt{\kappa_{1}} n_{1}^{L}, \\
& \mathcal{L}_{2}=\sqrt{\kappa_{1}} n_{1}^{R}, \\
& \mathcal{L}_{3}=\sqrt{\kappa_{2}} n_{2}^{L}, \\
& \mathcal{L}_{4}=\sqrt{\kappa_{2}} n_{2}^{R},
\end{aligned}
$$

where $\kappa_{1}$ and $\kappa_{2}$ are the phase-damping rates, which are proportional to the number density of noncondensed atoms.

The dynamics of the master equation is studied in terms of stochastic trajectories of state vectors [40, 41. In this approximation the density matrix of the system is written as the average of the stochastic wavefunctions:

$$
\rho(t)=\frac{1}{M C} \sum_{i=1}^{M C}\left|\Phi^{(i)}(t)\right\rangle\left\langle\Phi^{(i)}(t)\right|,
$$

where $\left|\Phi^{(i)}(t)\right\rangle$ is the $i$ th stochastic trajectory of the state vector, and $M C$ is the number of the simulations. In the evolution of the system two distinct elements can eventuate for small $\Delta t$. Either one of the quantum jumps 
occurs (with the probablity $\mathrm{d} p_{\alpha}=\left\langle\mathcal{L}_{\alpha}^{\dagger} \mathcal{L}_{\alpha}\right\rangle \Delta t$ ) which can be represented as an action of the corresponding Lindblad operator on the state of the system

$$
|\Phi\rangle \longmapsto \mathcal{L}_{\alpha}|\Phi\rangle,
$$

or the wavefunction is evolved by a non-Hermitian effective Hamiltonian (with the probablity $1-\sum_{\alpha} \mathrm{d} p_{\alpha}$ ), which has the form:

$$
H_{\text {eff }}=H-\frac{\mathrm{i} \hbar}{2} \sum_{k=1}^{4} \mathcal{L}_{k}^{\dagger} \mathcal{L}_{k} .
$$

The trajectories must be normalized after every time step. The Monte-Carlo approach has many advantages. Calculating a set of trajectories is more economical, than solving the master equation with a Runge-Kutta method. Moreover, a single-stochastic trajectory can be interpreted as a single realization of an experiment manipulating a unique quantum system [42].

Our calculation will be performed with parameter set $a_{11}=2 \mathrm{~nm}, a_{12}=4 \mathrm{~nm}, a_{22}=3 \mathrm{~nm}, N_{1}=5$, $N_{2}=20$ and phase damping rates $\kappa_{1}=\kappa_{2}=0.005$. The density matrix is obtained by averaging $M C=$ 5000 trajectories. Initially, among the 126 eigenstates there are about 60 cat states as Fig. 3(b) suggests. From these we shall investigate only two which can be written to a good approximation as $\left(|1,4\rangle_{1}|20,0\rangle_{2}+\right.$ $\left.|4,1\rangle_{1}|0,20\rangle_{2}\right) / \sqrt{2}$ (called the 61 th excited state) and $\left(|2,3\rangle_{1}|7,13\rangle_{2}+|3,2\rangle_{1}|13,7\rangle_{2}\right) / \sqrt{2}$ (the 63 th excited state) using the standard notation of the expansion (17).

Fig. 6 6 shows the magnitude of the density matrix of the 61th excited (cat) state at different times. At initial time $t=0$ there are two off-diagonal elements which are equal to the two diagonal ones. While the diagonal elements do not change significantly in time, the off-diagonal ones fall off gradually, and the state becomes a classical mixture after 2 second. A similar behavior (not shown) can be observed for the 63th cat state (and for all the other cat states).

To study finer details of the decoherence effect of environment on cat states we have calculated both $\operatorname{Tr} \rho^{2}$ and the magnitude $C$ of the average of the two maximal off-diagonal elements as function of time for the selected 61th and 63th excited states. These two quantities are characteristic of time development of the decoherence process, measuring formation of statistical mixture from the initital pure cat state.

The results are shown in Figs. 7 and 8 and we can observe that the decoherence is more effective for the state where the difference $\Delta N_{i}=\left|N_{i}^{L}-N_{i}^{R}\right|$ of particle numbers of the two components $i=1,2$ in the left and right potential well is greater. For example, in case of the 61th excited cat state, $\Delta N_{1}=3$ and $\Delta N_{2}=20$ which is greater than the corresponding values $\Delta N_{1}=1$ and $\Delta N_{2}=6$ of the 63 th state, and thus $\operatorname{Tr} \rho^{2}$ of the 61 th state approaches more quickly the value 0.5 , characteristic of the statistical mixture than that of the 63th state.

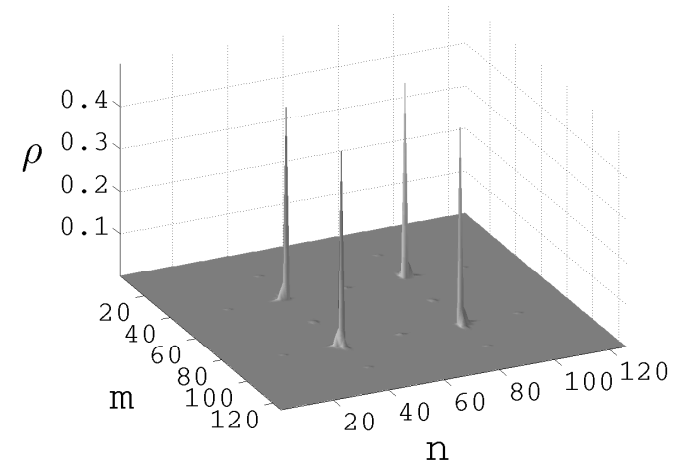

(a)

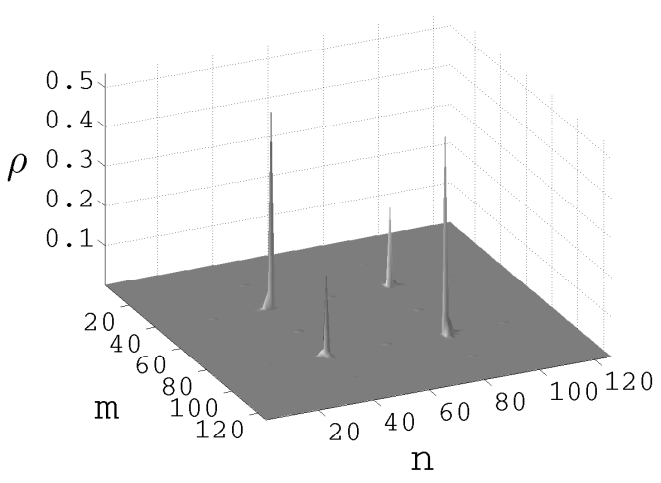

(b)

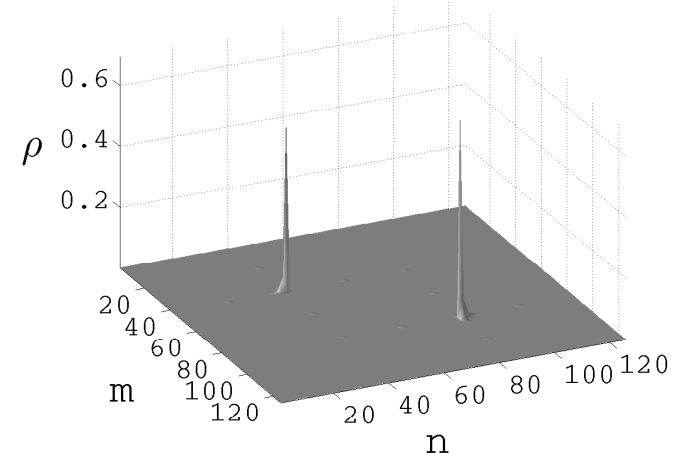

(c)

FIG. 6: The magnitude of density-matrix at different times (61th excited state): (a) initial state, (b) $t=0.5 \mathrm{sec}$, (c) $t=2 \mathrm{sec}$. Fixed parameters: $a_{11}=2 \mathrm{~nm}, a_{12}=4 \mathrm{~nm}$, $a_{22}=3 \mathrm{~nm}, N_{1}=5, N_{2}=20, \kappa_{1}=\kappa_{2}=0.005$.

A similar behavior can be observed when the time development of the average $C$ of the two maximal off-diagonal density matrix elements are studied in Figs. 7 and 8 . 


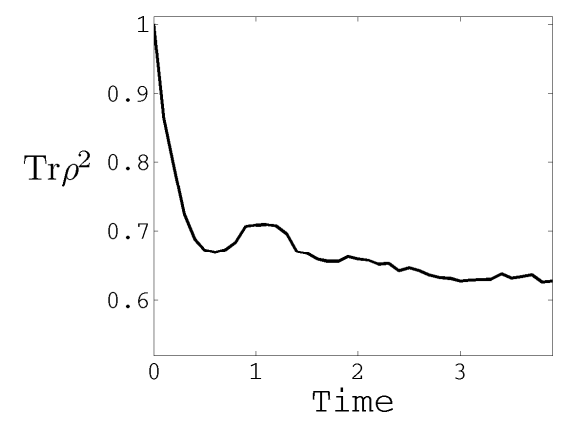

(a)

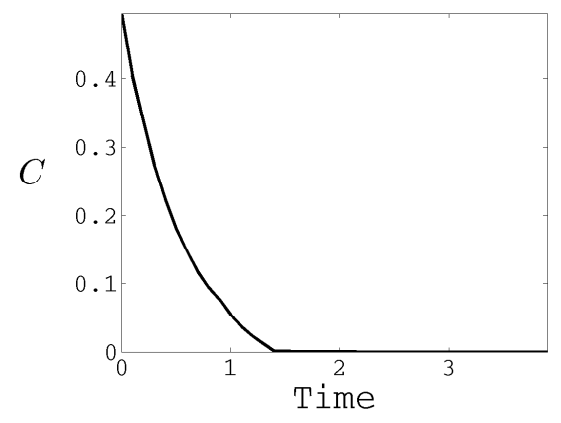

(b)

FIG. 7: The 61th excited state: $\left(|1,4\rangle_{1}|20,0\rangle_{2}+\right.$ $\left.|4,1\rangle_{1}|0,20\rangle_{2}\right) / \sqrt{2}$. Fixed parameters: $a_{11}=2 \mathrm{~nm}, a_{12}=$ $4 \mathrm{~nm}, a_{22}=3 \mathrm{~nm}, N_{1}=5, N_{2}=20, \kappa_{1}=\kappa_{2}=0.005$. Time is measured in units of second. (a) $\operatorname{Tr} \rho^{2}$ as function of time. (b) The magnitude $C$ of the average of the off-diagonal elements as function of time.

\section{CONCLUSION}

We have studied macroscopic quantum superposition (cat) states formed in two-component Bose-Einstein condensates described within the two-mode approximation. An analysis of the limitations of this approximation can be found in Ref. 43. However, the two-mode approximation has been ascertained to describe many aspects of double well experiments 44. The model has been applied to BECs where the two components correspond to two internal hyperfine state of ${ }^{87} \mathrm{Rb}$ atoms. Because of limited computational capacity, relatively modest particle numbers between 1-20 has been investigated, but a systematic check revealed that the main features of forming the cat states depend mostly on the actual interaction strengths (characterized by scattering lengths $a_{i j}$ ) between atoms, and not on the actual numbers $N_{i}, i=1,2$. Therefore we have performed exploratory calculations in wide range of values of scattering lengths $a_{i j}, i, j=1,2$, both in the attraction and repulsion domain, and presented the results in case of some selected examples exhibiting the most important features of formation, duration, and ceasing of MQSS or cat states.

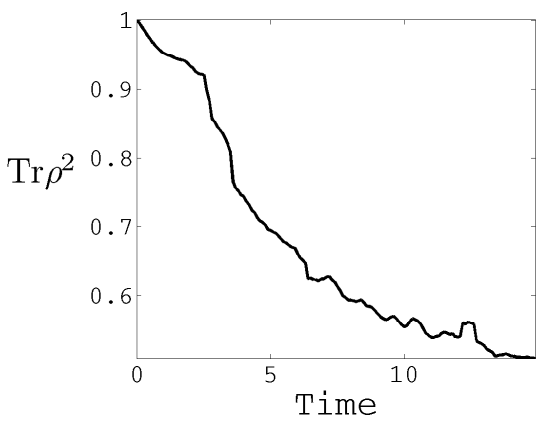

(a)

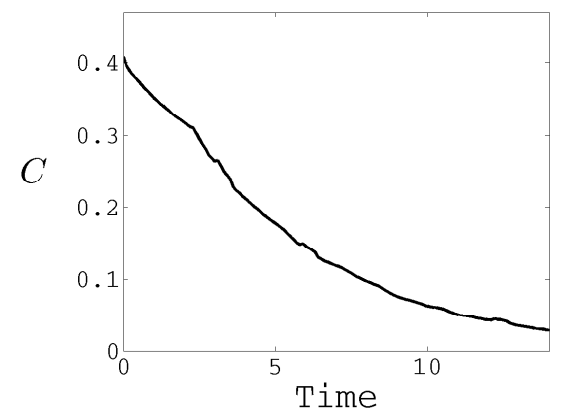

(b)

FIG. 8: The 63th excited state: $\quad\left(|2,3\rangle_{1}|7,13\rangle_{2}+\right.$ $\left.|3,2\rangle_{1}|13,7\rangle_{2}\right) / \sqrt{2}$. Fixed parameters: $a_{11}=2 \mathrm{~nm}, a_{12}=$ $4 \mathrm{~nm}, a_{22}=3 \mathrm{~nm}, N_{1}=5, N_{2}=20, \kappa_{1}=\kappa_{2}=0.005$. Time is measured in units of second. (a) $\operatorname{Tr} \rho^{2}$ as function of time. (b) The magnitude $C$ of the average of the off-diagonal elements as function of time.

We have found that, in general, positive inter-species scattering lengths $a_{i i}, i=1,2$ enhance the probability $P=N_{\text {cat }} / N_{\text {tot }}$ of two-component cat state formation. Small or zero intra-species scattering lengths $a_{12}$, of course, lessen this probability, as the system of twocomponent BEC actually decouples into two independent one-component BECs where, however, formation of onecomponent cat states is possible. In case of positive interspecies scattering length $a_{12}$ also the individual number $N_{\text {cat }}$ of two-component cat states is growing as it can be seen in Fig. 3(b) but negative $a_{12}$ hinders the formation of cat state as Fig. 4(b) shows. Finally, in the case when all the scattering lengths take negative values, there is only a limited range of two-component BEC cat state formation, studied both in terms of probability $P$ or the individual cat state number $N_{\text {cat }}$, as Figs. 5(a) and 5(b) exhibit.

Effect of environment has been modeled by phasedamping which is the result of elastic interaction between condensed and non-condensed atoms and preserves the particle numbers $N_{1}, N_{2}$. Using the corresponding Lindblad master equation (20) we have found that the most important parameter which gives rise to the decoher- 
ence effect of such type is the particle number difference $\Delta N_{i}=\left|N_{i}^{L}-N_{i}^{R}\right|, i=1,2$ between the left and right potential well. As Figs. 7 and 8 suggest the decoherence occurs more quickly for two-component cat states where the number difference $\Delta N_{i}$ is greater.

The influence of asymmetric wells, and other effects of the environment on the formation of cat states needs further research.

\section{Acknowledgment}

The authors thank Dr. Daniel Schumayer for reading the manuscript and providing valuable comments.
[1] W. H. Zurek, Physics Today 44, 36 (1991).

[2] W. H. Zurek, Rev. Mod. Phys. 75, 715 (2003).

[3] E. Schrödinger, Naturwissenschaften 23, 807 (1935).

[4] A. Bassi and G. Ghirardi, Physics Reports 379, 257 (2003).

[5] B. Yurke and D. Stoler, Phys. Rev. Lett. 57, 13 (1986).

[6] J. R. Friedman, V. Patel, W. Chen, S. K. Tolpygo, and J. E. Lukens, Nature 406, 43 (2000).

[7] M. Brune, E. Hagley, J. Dreyer, X. Maître, A. Maali, C. Wunderlich, J. M. Raimond, and S. Haroche, Phys. Rev. Lett. 77, 4887 (1996).

[8] J. M. Raimond, M. Brune, and S. Haroche, Rev. Mod. Phys. 73, 565 (2001).

[9] D. Leibfried, E. Knill, S. Seidelin, J. Britton, R. B. Blakestad, J. Chiaverini, D. B. Hume, W. M. Itano, J. D. Jost, C. Langer, et al., Nature 438, 639 (2005).

[10] A. Ourjoumtsev, H. Jeong, R. Tualle-Brouri, and P. Grangier., Nature 448, 784 (2007).

[11] I. Bloch, J. Dalibard, and W. Zwerger, Rev. Mod. Phys. 80, 885 (2008).

[12] C. A. Regal, M. Greiner, and D. S. Jin, Phys. Rev. Lett. 92, 040403 (2004).

[13] M. W. Zwierlein, C. A. Stan, C. H. Schunck, S. M. F. Raupach, A. J. Kerman, and W. Ketterle, Phys. Rev. Lett. 92, 120403 (2004).

[14] M. Greiner, O. Mandel, T. Esslinger, T. W. Hänsch, and I. Bloch, Nature 415, 39 (2002).

[15] Z. Hadzibabic, P. Krüger, M. Cheneau, B. Battelier, and J. Dalibard, Nature 441, 1118 (2006).

[16] D. Schumayer and D. A. Hutchinson, Phys. Rev. A 75, 015601 (2007).

[17] B. Paredes, A. Widera, V. Murg, O. Mandel, S. Fölling, I. Cirac, G. V. Shlyapnikov, T. W. Hänsch, and I. Bloch, Nature 429, 277 (2004).

[18] G. E. Astrakharchik, J. Boronat, J. Casulleras, and S. Giorgini, Phys. Rev. Lett. 95, 190407 (2005).

[19] J. I. Cirac, M. Lewenstein, K. Mølmer, and P. Zoller, Phys. Rev. A 57, 1208 (1998).

[20] D. Gordon and C. M. Savage, Phys. Rev. A 59, 4623 (1999).

[21] P. J. Y. Louis, P. M. R. Brydon, and C. M. Savage, Phys. Rev. A 64, 053613 (2001).

[22] J. Ruostekoski, M. J. Collett, R. Graham, and D. F. Walls, Phys. Rev. A 57, 511 (1998).

[23] K. W. Mahmud, H. Perry, and W. P. Reinhardt, Phys. Rev. A 71, 023615 (2005).
[24] E. Gross, Il Nuovo Cimento 20, 454 (1961).

[25] L. P. Pitaevskii, Soviet Phys.- JETP 13, 451 (1961).

[26] G. Csire, D. Schumayer, and B. Apagyi, Phys. Rev. A 82, 063608 (2010).

[27] A. Smerzi, S. Fantoni, S. Giovanazzi, and S. R. Shenoy, Phys. Rev. Lett. 79, 4950 (1997).

[28] A. Micheli, D. Jaksch, J. I. Cirac, and P. Zoller, Phys. Rev. A 67, 013607 (2003).

[29] D. Ananikian and T. Bergeman, Phys. Rev. A 73, 013604 (2006).

[30] I. I. Satija, R. Balakrishnan, P. Naudus, J. Heward, M. Edwards, and C. W. Clark, Phys. Rev. A 79, 033616 (2009).

[31] G. Mazzarella, M. Moratti, L. Salasnich, and F. Toigo, Journal of Physics B: Atomic, Molecular and Optical Physics 43, 065303 (2010).

[32] L. Lehtovaara, J. Toivanen, and J. Eloranta, Journal of Computational Physics 221, 148 (2007).

[33] J. Javanainen and J. Ruostekoski, Journal of Physics A: Mathematical and General 39, L179 (2006).

[34] W. H. Press, S. A. Teukolsky, W. T. Vetterling, and B. P. Flannery, Numerical Recipes in $\mathrm{C}++$ : The Art of Scientific Computing (Cambridge University Press, London, 2002).

[35] S. B. Papp, J. M. Pino, and C. E. Wieman, Phys. Rev. Lett. 101, 040402 (2008).

[36] C. Chin, R. Grimm, P. Julienne, and E. Tiesinga, Rev. Mod. Phys. 82, 1225 (2010).

[37] D. A. R. Dalvit, J. Dziarmaga, and W. H. Zurek, Phys. Rev. A 62, 013607 (2000).

[38] G. Lindblad, Communications in Mathematical Physics 48, 119 (1976).

[39] P. Talkner, Ann. Phys. (N.Y.) 167, 360 (1986).

[40] K. Mølmer, Y. Castin, and J. Dalibard, J. Opt. Soc. Am. B 10, 524 (1993).

[41] J. Steinbach, B. M. Garraway, and P. L. Knight, Phys. Rev. A 51, 3302 (1995).

[42] J. C. Bergquist, R. G. Hulet, W. M. Itano, and D. J. Wineland, Phys. Rev. Lett. 57, 1699 (1986).

[43] K. Sakmann, A. I. Streltsov, O. E. Alon, and L. S. Cederbaum, Phys. Rev. Lett. 103, 220601 (2009).

[44] M. Albiez, R. Gati, J. Fölling, S. Hunsmann, M. Cristiani, and M. K. Oberthaler, Phys. Rev. Lett. 95, 010402 (2005). 